\title{
A Correction Equation for Jump Height Measured Using the Just Jump System
}

\begin{tabular}{|r|l|}
\hline Journal: & International Journal of Sports Physiology and Performance \\
\hline Manuscript ID: & IJSPP.2015-0194.R2 \\
\hline Manuscript Type: & Brief Report \\
\hline Keywords: & biomechanics, physical performance \\
\hline \multicolumn{2}{|c}{} \\
\hline
\end{tabular}

\section{SCHOLARONE ${ }^{\mathrm{m}}$}

Manuscripts 


\section{1}

2

3

4

5

6

7

\title{
A Correction Equation for Jump Height Measured Using the Just Jump System
}

\author{
Submission Type: Brief Report
}

(7)

Authors: John J. McMahon, Paul A. Jones and Paul Comfort
Affiliations: McMahon, Jones and Comfort are with the Directorate of Sport, Exercise and Physiotherapy, University of Salford, Salford, UK
Corresponding Author: Address author correspondence to John J. McMahon at j.j.mcmahon@salford.ac.uk

\section{Preferred Running Head: Just Jump System: A Correction Equation}

Abstract Word Count: 250

Text-Only Word Count: 1639

Number of Tables: 0

\section{Number of Figures: 1}




\section{Abstract}

Purpose: The purpose of this study was to determine the concurrent validity and reliability of the popular Just Jump system (JJS) for determining jump height and, if necessary, provide a correction equation for future reference.

Methods: Eighteen male collegiate athletes performed three bilateral countermovement jumps (CMJs) on two JJSs ('alternative method') which were placed on top of a force platform ('criterion method'). Two JJSs were used to establish consistency between systems. Jump height was calculated from flight time obtained from the JJS and force platform, respectively.

Results: Intra-class correlation coefficients (ICCs) demonstrated excellent within-session reliability of the CMJ height measurement derived from both the JJS (ICC $=0.96, P<0.001$ ) and force platform $(\mathrm{ICC}=0.96, P<0.001)$. Dependent t-tests revealed that the JJS yielded a significantly greater $\mathrm{CMJ}$ jump height $(0.46 \pm 0.09 \mathrm{~m}$ vs. $0.33 \pm 0.08 \mathrm{~m})$ when compared to the force platform $(P<0.001$, Cohen's $d=1.39$, power $=1.00)$. There was, however, an excellent relationship between CMJ height derived from the JJS and force platform $(r=$ $0.998, P<0.001$, power $=1.00)$ with a coefficient of determination $\left(R^{2}\right)$ of 0.995 . Therefore, the following correction equation was produced: criterion jump height $=(0.8747 \times$ alternative jump height) -0.0666 .

Conclusions: The JJS provides a reliable, but an overestimated measure of jump height. It is suggested, therefore, that practitioners who use the JJS as part of future work should apply the correction equation presented in this study to resultant jump height values.

\section{Keywords: Countermovement jump, contact mat, concurrent validity, reliability}




\section{Introduction}

Vertical jump height provides an indices of lower limb power and as such, vertical jump testing of various athletic populations is commonplace among sports scientists and coaches. ${ }^{1}$ Many field-based assessments of jump height use contact mats, such as the Just Jump system (JJS) (Probotics Inc., Huntsville, AL, USA), which estimate jump height from flight time based on the following equation (1):

9

0

$$
J H=\frac{F T^{2} \times g}{8}
$$

Where $J H=$ jump height, $F T=$ flight time and $g=$ gravitational acceleration (i.e. $9.81 \mathrm{m.s} \mathrm{s}^{-2}$ )

The JSS was reported to provide a valid measurement of jump height based on derived results demonstrating similar values $(0.438 \pm 0.094 \mathrm{~m}$ vs. $0.442 \pm 0.103 \mathrm{~m}, p=0.972)$ to, and a high association $(r=0.967, p<0.01)$ with, jump height values derived from a three-camera motion capture system. ${ }^{2}$ Jump height was calculated by quantifying the peak height of a reflective marker placed on the subjects' sacrum relative to the initial height of the marker taken whilst subjects were standing as this was purported to be the gold standard method. ${ }^{2}$ The height of the center of mass (COM) at the instant of take-off needs to be taken into consideration, however, in order for accurate data to be determined via this method. Indeed, this notion has been addressed in studies which have used a linear position transducer to calculate vertical barbell displacement by zeroing the take-off height of the barbell whilst subjects were stood in a fully plantar flexed position. ${ }^{3}$ Not accounting for the vertical COM displacement achieved when subjects plantar flex their ankles would lead to an overestimation of jump height when using motion analysis and linear position transducers.

The JJS has been previously used to explore lower limb power differences between playing positions (forwards and backs) in elite junior rugby league players ${ }^{4}$ and associations between strength, sprint and jump performance in academy soccer players. ${ }^{5}$ As the JJS has been shown to yield reliable measures of jump height, ${ }^{6,7}$ the positional comparisons and performance associations explored by these researchers are acceptable, but the reported values may also be used as normative data. Accurate reporting of normative data is essential for allowing informed decisions regarding the prioritization of training goals to be made. Force platforms have been used as the criterion measure of jump height (derived from flight time) in recent studies which have validated the use of both alternative contact mat systems ${ }^{8}$ and iPhone apps ${ }^{9}$ alike due to their high sampling frequencies and accuracy in detecting movement and thus they may provide a more suitable comparison to the JJS.

The aim of this study was to determine the concurrent validity and reliability of the JJS for determining jump height (derived from flight time), with the same calculation applied using a force platform which acted as a criterion reference. It was hypothesized that there would be a strong positive relationship between the two jump height measures but that the values derived from the JJS would be significantly higher than those attained from the force platform. 


\section{Methods}

\section{Subjects}

Male collegiate athletes $(\mathrm{n}=18$, age $23.8 \pm 5.0$ years, body mass $78.7 \pm 10.9 \mathrm{~kg}$, height 1.77 $\pm 0.09 \mathrm{~m}$ ) from a variety of sports (e.g. soccer, rugby (both codes) and basketball), volunteered to participate in this study and provided written informed consent. The study was approved by the institutional ethics committee and conformed to the principles of the World Medical Association's Declaration of Helsinki (1983).

\section{Design}

This study employed a within-session repeated measures design whereby subjects attended a single testing session and performed multiple vertical jumps.

\section{Methodology}

Participants performed six bilateral countermovement jumps (CMJs), interspersed by two minutes of rest. They were instructed to perform a rapid eccentric phase, to approximately quarter squat depth, immediately followed a rapid concentric phase with the intention of jumping as high as possible. A qualified strength and conditioning coach visually ensured that each participant kept their arms akimbo throughout each jump and avoided flexing their lower limb joints during the flight phase.

Each CMJ was performed on a JJS placed directly on top of a 400-series strain gauge force platform (Fitness Technology, Adelaide, Australia) sampling at $600 \mathrm{~Hz}$. To ensure any differences in jump height observed between the JJS and the force platform were not due to a fault with the contact mat; participants performed the six CMJs on two separate JJSs (i.e. three jumps per system) in a randomized order.

Both flight time and jump height derived from the JJS and the 400 -series force platform were displayed on the attached hand-held computer and on custom software (Ballistic Measurement System, Fitness Technology, Adelaide, Australia), respectively. Both devices calculated jump height from flight time using equation 1. For the JJS, flight time was detected by the micro-switches embedded in the contact mat and for the 400 -series force platform, flight time was determined based on a vertical force threshold of $20 \mathrm{~N}$.

\section{Statistical Analysis}

Relative reliability of CMJ height attained between jump trials was determined using a twoway random-effects model intra-class correlation coefficient (ICC). The ICC values were interpreted according to previous work ${ }^{10}$ where a value of $\geq 0.80$ is considered highly reliable. Absolute reliability of between-trial CMJ height was assessed using the coefficient of variation $(\mathrm{CV})$. A dependent t-test was used to compare mean differences in CMJ values derived from the JJS and the force platform. Effect sizes were interpreted using the Cohen $d$ method which defines $0.2,0.5$, and 0.8 as small, medium, and large, respectively. ${ }^{11}$ Pearson correlation coefficient was used to determine the relationship between CMJ height derived from the two methods. Dependent t-tests and Pearson correlation coefficients were performed 
using SPSS software (version 20; SPSS Inc., Chicago, IL, USA). Post-hoc statistical power was calculated using $\mathrm{G}^{*}$ Power (version 3.1.9.2; University of Dusseldorf, Dusseldorf, Germany). ${ }^{12}$

\section{Results}

Within-session reliability of CMJ height was excellent when assessed using both the JJS and the force platform, with a comparable ICC value of $0.96(P<0.001)$ and CV values of $3.7 \%$ and $4.7 \%$, respectively. CMJ height derived from each JJS was identical to one another $(0.46$ $\pm 0.09 \mathrm{~m})$, but significantly greater $(P<0.001$, Cohen's $d=1.39$, power $=1.00)$ than values determined using the force platform $(0.33 \pm 0.08 \mathrm{~m})$.

There was a positive relationship between CMJ height derived from the JJS and the force platform $(r=0.998, P<0.001$, power $=1.00)$ which resulted in a coefficient of determination $\left(R^{2}\right)$ of 0.995 (Figure 1). Given the near perfect coefficient of determination observed between the two systems, a linear regression equation was established to allow vertical jump height values derived from the JJS to be corrected (Figure 1).

\section{INSERT FIGURE 1 ABOUT HERE}

\section{Discussion}

This study aimed to determine the concurrent validity of the JJS for determining jump height (derived from flight time) by comparing it to those calculated using the same method using a force platform (criterion reference). The main finding of this study was that the JJS overestimated CMJ height when compared to the force platform $(0.46 \pm 0.09 \mathrm{~m}$ vs. $0.33 \pm$ $0.08 \mathrm{~m}$ ), in line with the hypothesis; suggesting that the JJS does not provide a valid measure of jump height. This finding opposes those of an earlier study which validated the JJS against a three-camera motion capture system. ${ }^{2}$ As mentioned earlier, the aforementioned authors did not account for the effects of plantar flexion demonstrated prior to the take-off phase of vertical jumping which subsequently led to an overestimation of jump height. The JSS may have overestimated $\mathrm{CMJ}$ height due to the associated hardness of contact mats requiring a large minimum force (perhaps $>20 \mathrm{~N}$ ) in order to activate its mechanical circuit and detect landing. ${ }^{8}$

Despite the large mean difference in jump height, there was an excellent relationship between the two systems $(r=0.998)$ with values from the JJS able to explain $99.5 \%$ of the variance of values from the force platform (Figure 1). This is useful in that jump height values determined using the JJS can be subsequently corrected based on the following equation (2): 


$$
C J H=(0.8747 * A J H)-0.0666
$$

$$
\text { Where } C J H=\text { criterion jump height }(m) \text { and AJH = alternative jump height }(m)
$$

In addition to demonstrating a high association with data derived from the force platform, CMJ height derived from the JJS also yielded identical within-session reliability (ICC = 0.96). This finding is in line with previous work ${ }^{6,7}$ and suggests that the JJS can be utilized as a reliable field-based method of determining jump height. Based on reliability data, it is viable that the JJS can be used to monitor changes in jump height in future studies, despite the attained values being inflated in comparison to the criterion measure, by applying equation 2 to the data.

When interpreting the results of this study, it should be noted that previous studies found that the flight time method (equation 1) overestimated CMJ height (by approximately 3\% in males ${ }^{13}$ ) when compared to the-preferred velocity at take-off method. ${ }^{13,14}$ This error in jump height estimation was attributed to postural changes during the flight phase of the jumps (e.g. tucking the legs) ${ }^{13}$, however, it is important to note that the present study did not include any $\mathrm{CMJ}$ trials that were performed in this manner and thus any associated error may considered negligible.

\section{Practical Applications}

It is suggested that practitioners who use the JJS should apply equation 2 to all future data in order correct their values. Equation 2 can also be applied to previous research which has used the JJS to calculate jump height. This would bring the jump height values attained in those studies in line with the criterion reference and thus provide practitioners with the ability to interpret this data more accurately.

\section{Conclusions}

The JJS is reliable, but overestimates jump height when compared to the flight time-derived jump height obtained from the force platform.

\section{References}

1. Klavora P. Vertical-jump tests: a critical review. Strength \& Conditioning Journal. 2000;22(5):70-74.

2. Leard JS, Cirillo MA, Katsnelson E, et al. Validity of two alternative systems for measuring vertical jump height. The Journal of Strength \& Conditioning Research. 2007;21(4):1296-1299.

3. Thomasson ML, Comfort P. Occurrence of fatigue during sets of static squat jumps performed at a variety of loads. The Journal of Strength \& Conditioning Research. 2012;26(3):677-683.

4. Kirkpatrick J, Comfort P. Strength, power, and speed qualities in english junior elite rugby league players. The Journal of Strength \& Conditioning Research. 2013;27(9):2414-2419. 
252

253

254

255

256

257

258

259

260

261

262

263

264

265

266

267

268

269

270

271

272

273

274

275

276

277

278

279

280

281

282

283

284

5. Comfort P, Stewart A, Bloom L, Clarkson B. Relationships between strength, sprint, and jump performance in well-trained youth soccer players. The Journal of Strength \& Conditioning Research. 2014;28(1):173-177.

6. Nuzzo JL, Anning JH, Scharfenberg JM. The reliability of three devices used for measuring vertical jump height. The Journal of Strength \& Conditioning Research. 2011;25(9):2580-2590.

7. Moir G, Shastri P, Connaboy C. Intersession reliability of vertical jump height in women and men. The Journal of Strength \& Conditioning Research. 2008;22(6):1779-1784.

8. García-López J, Morante JC, Ogueta-Alday A, Rodríguez-Marroyo JA. The type of mat (contact vs. photocell) affects vertical jump height estimated from flight time. The Journal of Strength \& Conditioning Research. 2013;27(4):1162-1167.

9. Balsalobre-Fernández C, Glaister M, Lockey RA. The validity and reliability of an iPhone app for measuring vertical jump performance. Journal of Sports Sciences. 2015/03/12 E-Pub:1-6.

10. Cortina JM. What is coefficient alpha? An examination of theory and applications. Journal of Applied Psychology. 1993;78(1):98-104.

11. Rhea MR. Determining the magnitude of treatment effects in strength training research through the use of the effect size. The Journal of Strength \& Conditioning Research. 2004;18(4):918-920.

12. Faul F, Erdfelder E, Buchner A, Lang AL. Statistical power analyses using G*Power 3.1: Tests for correlation and regression analyses. Behavior Research Methods. 2009;41(4):1149-1160.

13. Moir GL. Three Different Methods of Calculating Vertical Jump Height from Force Platform Data in Men and Women. Measurement in Physical Education and Exercise Science. 2008;12(4):207-218.

14. Aragón LF. Evaluation of Four Vertical Jump Tests: Methodology, Reliability, Validity, and Accuracy. Measurement in Physical Education and Exercise Science. 2000;4(4):215-228.

\section{Figures}




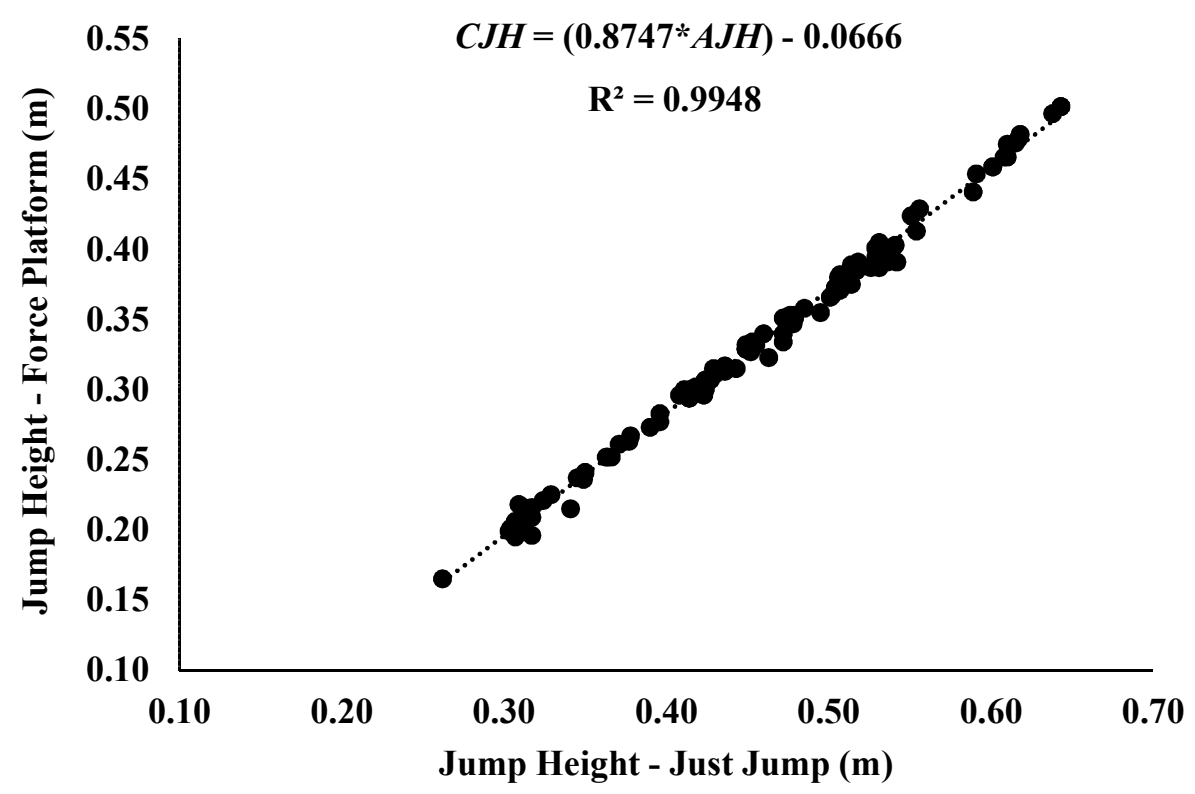

Figure 1: Relationship between vertical jump height values derived from the Just Jump system and the force platform for pooled data taken from the six $(n=108)$ jump trials (where $\mathrm{CJH}=$ criterion jump height and $\mathrm{AJH}=$ alternative jump height). 